\title{
Cytomegalovirus disease in immunocompetent adults
}

Daniel Lancini MBBS Student

Helen M Faddy

$\mathrm{BSc}$ (Hons), PhD Senior Research Fellow, Research and Development ${ }^{2}$

Robert Flower $\mathrm{PhD}$ Research Program Leader, Research and Development ${ }^{2}$

Chris Hogan

MBBS, BSc(Hons), FRCPA Medical Director Pathology Services ${ }^{2}$

1 School of Medicine, University of Queensland, Brisbane, QLD.

2 Australian Red Cross Blood Service, Brisbane, QLD.

daniel.lancini@ uqconnect.edu.au

doi: 10.5694/mjal4.00183
C

ytomegalovirus (CMV) is an internationally ubiquitous human herpes virus with a worldwide seroprevalence ranging from $45 \%$ to $100 \%{ }^{1}$ A national serosurvey in 2006 estimated that $57 \%$ of Australians between the ages of 1 and 59 years were seropositive. ${ }^{2}$ While primary CMV infection is common in the general community, it is usually asymptomatic or causes a mild mononucleosis-like syndrome. ${ }^{3}$ The viraemic phase is generally self-limiting in healthy adults, and is followed by a lifelong bloodborne latent phase within peripheral monocytes and CD34+ myeloid progenitor cells ${ }^{4}$ (Box 1).

However, in certain circumstances CMV infection is capable of producing severe, life-threatening disease, including a wide range of potential clinical manifestations, owing to systemic haematogenous dissemination and a very broad tissue tropism ${ }^{6}$ (Box 2). Typically, severe CMV disease occurs in the context of an immature, suppressed or compromised immune system, and can lead to death or permanent major sequelae. ${ }^{7}$ As such, severe CMV infection is a well recognised cause of morbidity and mortality in neonates and immunocompromised adults, such as pharmacologically immunosuppressed transplant recipients and patients with AIDS. ${ }^{7}$

\section{CMV disease in immunocompetent adults}

While CMV is a well recognised pathogen in neonates and immunocompromised adults, the burden of CMV disease in immunocompetent adults is less well understood. This is because severe CMV disease is of considerably lower incidence in this population. However, it is far from non-existent; over 380 published case reports document instances of severe tissue-invasive CMV infection in immunocompetent adults. Similarly to CMV disease in immunocompromised individuals, these cases show a wide range of manifestations, including colitis, ${ }^{9}$ vascular thrombosis, ${ }^{10}$ pneumonia ${ }^{11}$ and myocarditis. ${ }^{12}$

The most comprehensive evaluation of severe CMV infection in immunocompetent adults to date included a systematic meta-analysis of case reports and reviews documenting 290 instances, across all manifestations. ${ }^{8}$ This study found that CMV infection most commonly involved the gastrointestinal tract (primarily colitis), followed by the central nervous system (including meningitis, encephalitis and myelitis) and then haematological abnormalities (including haemolytic anaemia and thrombocytopenia). CMV disease of the eye, liver, lung and vasculature were also documented, among other conditions. The authors ultimately concluded that the incidence of severe manifestations of CMV infection in immunocompetent individuals appeared to be significantly more common than previously appreciated. ${ }^{8}$

\section{Summary}

Cytomegalovirus (CMV) is a highly prevalent and globally distributed virus.

CMV infection in healthy adults is usually asymptomatic or causes a mild mononucleosis-like syndrome.

- CMV disease causes significant morbidity and mortality in neonates and severely immunocompromised adults.

- CMV disease can present with a wide range of manifestations, with colitis being the most common.

The incidence of severe CMV disease in immunocompetent adults appears to be greater than previously thought, which may be partly due to immune dysfunction related to comorbidities such as kidney disease or diabetes mellitus.

CMV disease can mimic an array of alternative diagnoses and pose a significant diagnostic challenge, especially in immunocompetent adults, leading to delayed diagnosis, adverse health outcomes and unnecessary financial expense.

Non-invasive testing for CMV is widely available and can facilitate early diagnosis if used appropriately.

Although limited, current evidence suggests that targeted antiviral therapy with ganciclovir or valganciclovir is appropriate for severe CMV disease in immunocompetent adults.

It should be noted that the definition of immunocompetency in this analysis, like most published reviews and case reports, excluded only individuals with profound loss of immune function, including patients who had AIDS, pharmacologically immunosuppressed transplant recipients and chemotherapy recipients. However, many case reports of CMV disease in "immunocompetent" adults document comorbidities that may be associated with a degree of immune dysfunction, such as diabetes mellitus or renal failure. Indeed, studies have also shown an increased risk of CMV-related morbidity and mortality in "immunocompetent" critically ill patients. ${ }^{13}$ It is therefore highly feasible that partial immune dysfunction may represent a currently overlooked risk factor for severe CMV disease. As such, further studies are needed to evaluate the risk of CMV disease in these populations. Nonetheless, this possibility reinforces the importance of considering CMV as a potential infectious agent even in patients with a low degree of immune dysfunction.

\section{Diagnostic challenge of CMV disease}

Although uncommon, severe CMV infection in immunocompetent adults often poses a significant diagnostic challenge, and a number of case reports have documented 


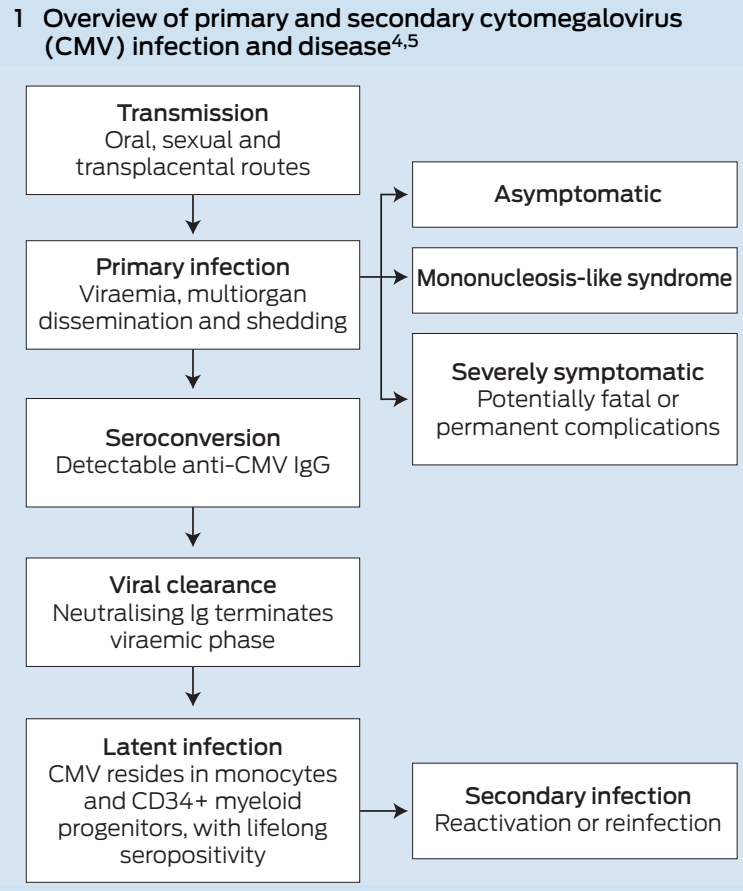

considerable delay to diagnosis. ${ }^{14-16}$ Some patients with CMV colitis, in particular, have had protracted hospitalisations and have undergone surgery to investigate persistent and undiagnosed disease.

The diagnostic difficulty in CMV disease arises from three factors. First, the low incidence of severe CMV disease in immunocompetent individuals warrants a lower index of clinical suspicion for CMV infection at initial presentation. Second, CMV disease can present with a wide array of potential clinical manifestations, owing to broad tissue tropism. Third, certain presentations of CMV disease strongly mimic other diseases, potentially causing diagnostic confusion and delay in diagnosis. Indeed, case studies have documented initial misdiagnoses of colon carcinoma, ${ }^{14}$ ischaemic colitis ${ }^{15}$ inflammatory bowel disease, ${ }^{16}$ dengue fever ${ }^{17}$ and lung cancer, ${ }^{18}$ among others.

\section{Notable case studies}

Siegal and colleagues documented the case of an 82-yearold man presenting with a 2-day history of diarrhoea and epigastric pain. ${ }^{15}$ Non-contrast computed tomography (CT) showed faecal impaction and thickening of the wall of the distal colon, with generalised large-bowel dilatation. Despite repeated negative results from assays for Clostridium difficile toxin, antibiotics were administered. There was no clinical improvement. Sigmoidoscopy and biopsy during the second week of admission showed acute inflammation and lymphoid aggregates, but did not lead to a diagnosis. The patient was treated with mesalamine for suspected ulcerative colitis, with minimal effect. A positive faecal occult blood test result raised the suspicion of ischaemic colitis. However, repeat sigmoidoscopy and biopsy at 1 month after admission showed mucosal ulceration with viral inclusions diagnostic of CMV infection, and treatment with intravenous (IV) ganciclovir was

2 Non-exhaustive list of recognised potential
manifestations of cytomegalovirus disease
Direct effects

commenced. The authors concluded that CMV should be considered as a potential aetiological agent of severe colitis in immunocompetent individuals when other differentials have been excluded. ${ }^{15}$

A case study by Falagas and colleagues highlighted the diagnostic difficulty posed by CMV disease in non-immunocompromised adults. ${ }^{14}$ In this instance, a 57-year-old, HIV-negative man with chronic renal failure presented with acute abdominal pain, diarrhoea, and per rectal bleeding. Colonoscopy showed a large polypoid mass in the hepatic flexure, suspicious for colorectal carcinoma, although a colonic biopsy specimen did not show neoplastic changes. The results of subsequent CT scanning, stool examination and a $C$. difficile toxin assay were normal. Recurrent symptoms prompted an exploratory laparotomy and right hemicolectomy on Day 9 of admission, at which time histological analysis showed intranuclear inclusions diagnostic of CMV disease. The patient commenced a 2-week course of IV ganciclovir, and his symptoms subsequently abated. The authors concluded that the endoscopic findings of CMV colitis may resemble colon carcinoma and should be considered as a differential diagnosis, even in patients without severe immunosuppression. ${ }^{14}$

$\mathrm{Yu}$ and colleagues published a case report of a 39-yearold woman with a 6-week history of fever of unknown origin who was referred to a tertiary hospital. ${ }^{19}$ Her liver enzyme levels were elevated, and an abdominal CT scan was consistent with acute hepatitis. Results of serological screening for hepatitis A, B and C viruses, Epstein-Barr virus and HIV were negative. The result of a CMV-polymerase chain reaction (PCR) analysis was positive, and other causes (including drug-induced and autoimmune) were excluded. The severity and progressive 
nature of the disease necessitated urgent living-donor liver transplantation. A biopsy specimen from her explant liver showed widespread hepatic necrosis and stained positive for CMV protein. Initially, no antiviral therapy was commenced. The patient's postoperative course was characterised by a rising serum bilirubin level and CMV antigenaemia. A liver biopsy specimen taken on Day 14 after the operation showed moderate degenerative changes and stained positive for CMV protein, at which point IV ganciclovir therapy was initiated, leading to improvement in her clinical condition and liver function. The authors concluded that CMV should be investigated as a potential cause of severe hepatitis, regardless of the patient's immune status, after more common aetiologies have been excluded. ${ }^{19}$

\section{Clinical implications}

Delayed diagnosis of CMV disease in immunocompetent adults creates the potential for numerous adverse outcomes. Delay in initiation of targeted therapy leads to increasing morbidity and mortality as a result of disease progression. Prolonged hospitalisation is associated with health risks such as nosocomial infection and venous thromboembolism. Patients may also receive unnecessary radiation exposure from repeated $\mathrm{CT}$ imaging and be exposed to risks associated with surgical interventions. In addition, financial costs associated with extended hospitalisation and the potential for numerous investigations, surgery and intensive care unit admission are substantial. These consequences of diagnostic delay are of particular note, given the availability of non-invasive diagnostic testing for CMV infection, including serological tests, CMV-PCR and viral culture. ${ }^{20}$

\section{Treatment of CMV disease in immunocompetent adults}

While ganciclovir or valganciclovir are currently recommended as first-line treatment for severe CMV disease in immunocompromised adults, few studies have appropriately evaluated the use of these antiviral agents for the treatment of severe CMV disease in immunocompetent adults. These agents may have major side effects, including myelosuppression and potential carcinogenicity. However, untreated CMV disease is associated with considerable morbidity and mortality, and published case studies and reviews provide consistent case-based evidence of rapid clinical improvement after commencement of therapy in this clinical setting. $3,11,17,21-23$ Furthermore, a recent study found ganciclovir to be a safe and effective treatment for CMV-associated pneumonia in immunocompetent children. ${ }^{24}$ Therefore, the continued use of antivirals for the treatment of very likely or proven CMV disease in immunocompetent adults appears justified at present. While formal studies evaluating the efficacy and utility of these therapies in the context of immunocompetency would be beneficial, such studies would be difficult to pursue, given the low incidence of severe CMV disease in this population. ${ }^{3}$

\section{Conclusion}

Although severe CMV disease primarily occurs in neonates or severely immunocompromised adults, the burden of disease in immunocompetent adults appears to be greater than previously understood. This may be partly owing to underrecognised risk from immune dysfunction associated with comorbidities such as renal failure or diabetes mellitus. It also appears that diagnostic delay is more likely in this clinical setting, especially for instances of CMV colitis, creating the potential for a range of adverse outcomes. Severe CMV disease in immunocompetent adults is likely to remain a diagnostic challenge in many circumstances. However, earlier consideration of CMV as a potential aetiological agent in individuals with atypical or refractory disease, regardless of immune status, may facilitate early non-invasive diagnosis and the initiation of appropriate directed antiviral therapy.

Acknowledgements: We acknowledge Australian governments that fully fund the Australian Red Cross Blood Service to provide blood products and services to the Australian community.

Competing interests: No relevant disclosures.

Provenance: Not commissioned; externally peer reviewed.

1 Cannon MJ, Schmid DS, Hyde TB. Review of cytomegalovirus seroprevalence and demographic characteristics associated with infection. Rev Med Virol 2010; 20: 202-213.

2 Seale H, Maclntyre CR, Gidding HF, et al. National serosurvey of cytomegalovirus in Australia. Clin Vaccine Immunol 2006; 13: 1181-1184.

3 Eddleston M, Peacock S, Juniper M, Warrell DA. Severe cytomegalovirus infection in immunocompetent patients. Clin Infect Dis 1997; 24: 52-56.

4 Sinclair J. Human cytomegalovirus: latency and reactivation in the myeloid lineage. J Clin Virol 2008; 41: 180-185.

5 Ziemann M, Heuft HG, Frank K, et al. Window period donations during primary cytomegalovirus infection and risk of transfusion-transmitted infections. Transfusion 2013; 53: 1088-1094.

6 Sinzger C, Digel M, Jahn G. Cytomegalovirus cell tropism. Curr Top Microbiol Immunol 2008; 325: 63-83.

7 Griffiths PD. Burden of disease associated with human cytomegalovirus and prospects for elimination by universal immunisation. Lancet Infect Dis 2012; 12: 790-798.

8 Rafailidis PI, Mourtzoukou EG, Varbobitis IC, Falagas ME. Severe cytomegalovirus infection in apparently immunocompetent patients: a systematic review. Virol J 2008; 5: 47.

$9 \mathrm{Seo} \mathrm{TH}, \mathrm{Kim} \mathrm{JH}$, Ko SY, et al. Cytomegalovirus colitis in immunocompetent patients: a clinical and endoscopic study. Hepatogastroenterology 2012; 59: 2137-2141.

10 Abgueguen P, Delbos V, Chennebault JM, et al. Vascular thrombosis and acute cytomegalovirus infection in immunocompetent patients: report of 2 cases and literature review. Clin Infect Dis 2003; 36: El34-El39.

11 Grilli E, Galati V, Bordi L, et al. Cytomegalovirus pneumonia in immunocompetent host: case report and literature review. J Clin Virol 2012; 55: 356-359.

12 Vanstechelman F, Vandekerckhove H. Cytomegalovirus myocarditis in an immunocompetent patient. Acta Cardiol 2012; 67: 257-260.

13 Osawa R, Singh N. Cytomegalovirus infection in critically ill patients: a systematic review. Crit Care 2009; 13: R68.

14 Falagas ME, Griffiths J, Prekezes J, Worthington M. Cytomegalovirus colitis mimicking colon carcinoma in an HIV-negative patient with chronic renal failure. Am J Gastroenterol 1996; 91: 168-169.

15 Siegal DS, Hamid N, Cunha BA. Cytomegalovirus colitis mimicking ischemic colitis in an immunocompetent host. Heart Lung 2005; 34: 291-294.

16 Rezania D, Ouban A, Marcet J, et al. CMV colitis mimicking recurrent inflammatory bowel disease: report of three cases. Am Surg 2007; 73: 58-61.

17 Tirumala S, Behera B, Lingala S, et al. Severe cytomegalovirus infections in immunocompetent patients at admission as dengue mimic: successful treatment with intravenous ganciclovir. Asian Pac J Trop Med 2012; 5: $920-922$.

18 Karakelides H, Aubry MC, Ryu JH. Cytomegalovirus pneumonia mimicking lung cancer in an immunocompetent host. Mayo Clin Proc 2003; 78: 488-490.

19 Yu YD, Park GC, Park PJ, et al. Cytomegalovirus infection-associated fulminant hepatitis in an immunocompetent adult requiring emergency living-donor liver transplantation: report of a case. Surg Today 2013; 43: 424-428.

20 Kalkan IH, Da li U. What is the most accurate method for the diagnosis of cytomegalovirus (CMV) enteritis or colitis? Turk J Gastroenterol 2010; 21: 83-86.

21 Snape SE, Venkatesan P. Valganciclovir treatment of primary cytomegalovirus pneumonitis in an immunocompetent adult. BMJ Case Rep [internet] 2011; 2011. pii: bcrl120103489.

22 Leman JS, Toalson TW, Wlodaver CG. Ganciclovir treatment of systemic cytomegalovirus in an immunocompetent host. J Okla State Med Assoc 1997; 90: 367-369.

23 Serna-Higuera C, González-García M, Milicua JM, Muñoz V. Acute cholestatic hepatitis by cytomegalovirus in an immunocompetent patient resolved with ganciclovir. J Clin Gastroenterol 1999; 29: 276-277.

24 Doan TT, Phung TT, Pham HV, et al. Effect of ganciclovir for the treatment of severe cytomegalovirusassociated pneumonia in children without a specific immunocompromised state. BMC Infect Dis 2013: 13: 424. 